\title{
FAMILIAL BENIGN CHRONIC PEMPHIGUS (HAILEY-HAILEY DISEASE)
}

\author{
Ivelina Yordanova, Dimitar Gospodinov, Valentina Chavdarova, Savelina \\ Popovska*, \\ Department of Dermatology and Venereology, \\ *Department of General and Clinical Pathology, \\ Medical University - Pleven, Bulgaria
}

\section{SUMMARY:}

Familial benign chronic pemphigus (Hailey-Hailey disease) is a blistering dermatosis, which is inherited as an autosomal dominant trait and usually presents around the third and fourth decades. Painful erosions, vesico-pustules and scaly erythematous plaques appear at sites of friction such as the sides of the neck, the axillae, the groins and the perineum. A case of familial benign chronic pemphigus in a 54 year-old woman is presented. The disease started at the age of 35. The areas of predilection are the axillary and submammary folds and genital area. Erythematous, macerated plaques were found with multiple painful fissures, vesicles and crusts in its periphery. The diagnosis was established on the basis of physical examination and was confirmed by histological examination of a skin biopsy. A suprabasal cell separation (acantholysis) of the epidermis was found. A family history is present. An autosomal dominant mode of inheritance was confirmed by genealogical analysis. Thirteen members have been affected by the same disease. In the presented case topical and systemic antibiotics, antimycotics, corticosteroids and retinoids were applied with a transient result. The patient was treated with topical Pimecrolimus which showed a good result. Postlesional hyperpigmentations were found after this treatment.

Key words: Familial benign chronic pemphigus, Hailey-Hailey disease, acantholysis, Pimecrolimus.

\section{INTRODUCTION:}

Familial benign chronic pemphigus (Hailey-Hailey disease) is a blistering dermatosis, which is inherited as an autosomal dominant trait and usually presents itself around the third or fourth decades. Painful erosions, vesico-pustules and scaly erythematous plaques appear on the sites of friction such as the neck, axillae, groins and perineum.

\section{Case report:}

A 54-year-old woman with a nineteen-year history of flexural blistering eruptions was presented. The disease started at the age of 35 with recurrent erythema, vesicles and erosions in the intertriginous areas - axillary, submammary, inguinal folds and the neck. Bullous and erosive lesions epithelised slowly without scars. There was no seasonal influence, however the skin eruptions appeared around menstruation. The patient's father and other 11 relatives in three consequent generations were affected by the same disease. Physical examination showed erythematous, macerated plaques with multiple fissures, peripheral vesicles and crusts in the axillary and submammary folds and the genital area (Fig.1). The routine blood count and urine analysis were within the normal limits. Microbiological examination revealed Candida albicans and Staphylococcus aureus. Histological examination of skin biopsy showed intraepidermal clefts of varying sizes both suprabasally and higher in the epidermis, as well as the characteristic incomplete acantholysis giving it the appearance of a "dilapidated brick wall" (Fig. 2). An autosomal dominant mode of inheritance was confirmed by genealogical analysis. Thirteen family members have been affected by the same disease (fig. 3). The patient's medical history revealed a negative direct immunofluorescence evaluation, which is consistent with a diagnosis of Hailey-Hailey disease (or chronic benign familial pemphigus). In the presented case systemic and topical antibiotics (Clindamycin caps., Fucidin cream), antimycotics (Itraconazole), topical corticosteroids and retinoids were applied with a transient result. Finally the patient was treated with topical pimecrolimus two times a day with a good result. All of the lesions healed within 2 weeks. Postlesional hyperpigmentations were found after this treatment (fig. 4). We observed no side effects like pruritus and burning sensation. The patient was followed up.

\section{DISCUSSION:}

Hailey-Hailey disease, or chronic benign familial pemphigus, is a genodermatosis arising in adult age with recurrent vesicles and erosions primarily in the flexural areas. Along with the typical intertriginous localization of HaileyHailey disease, generalized cutaneous involvement may also occur $(6,10,14)$. It is an autosomal dominant skin disorder characterized by abnormal keratinocyte adhesion in the suprabasal layers of the epidermis. The responsible defect has been identified in the gene named ATP2C1 on 
chromosome 3q21-24, encoding the human secretory pathway $\mathrm{Ca}(2+)$-ATPase (hSPCA1) (9). More than 82 different ATP2C1 mutations have been described up to date. No direct genotype-phenotype correlation between a specific mutation and the disseminated variant of Hailey-Hailey disease has been demonstrated. Suction has been used to investigate cell adhesion in clinically normal skin in Hailey-Hailey disease.They have demonstrated that there is a widespread subclinical abnormality in keratinocyte adhesion in this disease. There may be a synthesis of functionally deficient adhesion junctions, increased breakdown of adhesion junctions or abnormalities in other adhesion proteins in the epidermis in Hailey-Hailey disease. The findings contrast with those in Darier's disease in which abnormal cell adhesion was only demonstrable in clinically involved skin. Although the clinical and histological pictures of these diseases overlap, characteristic clinical features make differentiation possible $(3,8,11)$.Familial benign chronic pemphigus (Hailey-Hailey disease) is often disabling genodermatosis that may not respond to conservative dermatologic therapy (7). It can be successfully treated by excision of the lesional skin followed by split-thickness grafting. The success of surgery is attributed to the removal of adnexal structures and a decrease in sweating and maceration. Surgical excision provided definite relief from an otherwise disabling disease and a satisfactory improvement in life-style $(1,4)$. This condition may be treated successfully by carbon dioxide (CO2) laserabrasion. The procedure spared the underlying adnexae which contributed to the reepithelialization of the epidermis (5). Treatment with systemic retinoids followed by aromatic retinoids was tried but eczema herpeticum developed in the affected areas and dissemination of Hailey-Hailey disease ultimately occurred (12). The strong steroid ointments are really effective for this disease when secondary infection was prevented by the application of antibiotics and/or antimycotics as in our case $(7,9)$. No serious side-effects except skin atrophy and contact dermatitis were observed. Morbus Hailey-Hailey may be treated with cyclosporin too (2).There was a clear improvement in the affected skin area. The response lasted for 24 weeks but there was a gradual deterioration after the treatment was stopped.Topical tacrolimus ointment is an effective therapy for Hailey-Hailey disease (13). We obtained similar results in the treatment with topical pimecrolimus.

IN CONCLUSION we present a case of familial benign chronic pemphigus (Hailey-Hailey disease), which started in the fourth decade with painful bullous and erosive lesions and scaly erythematous plaques which appeared at the sites of friction. In the presented case topical and systemic antibiotics, antimycotics, corticosteroids and retinoids were applied with a transient result. We have demonstrated a good effect with topical pimecrolimus treatment.

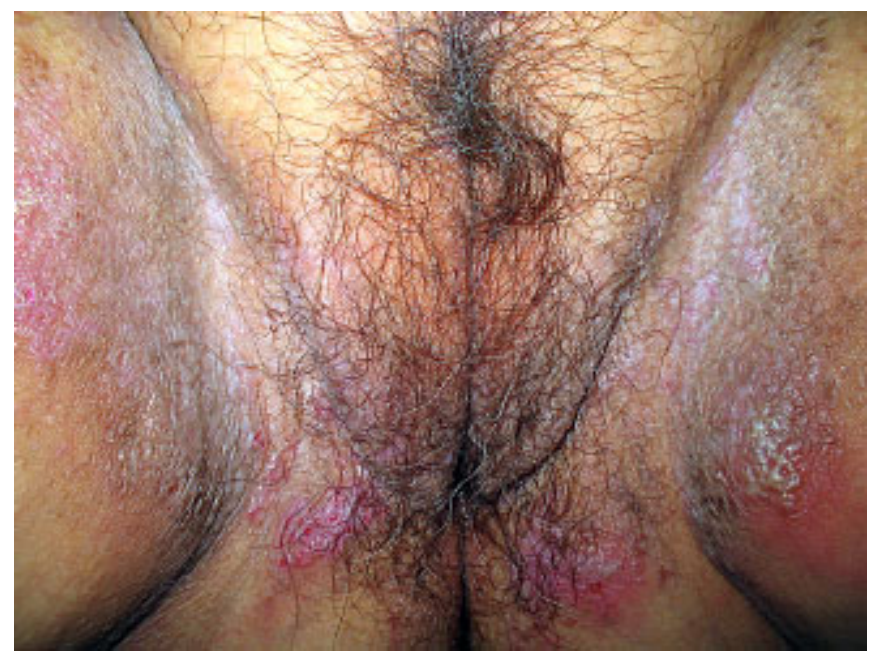

Fig. 1. Erythematous, macerated plaques with multiple peripheral vesicles and crusts in the genital area.

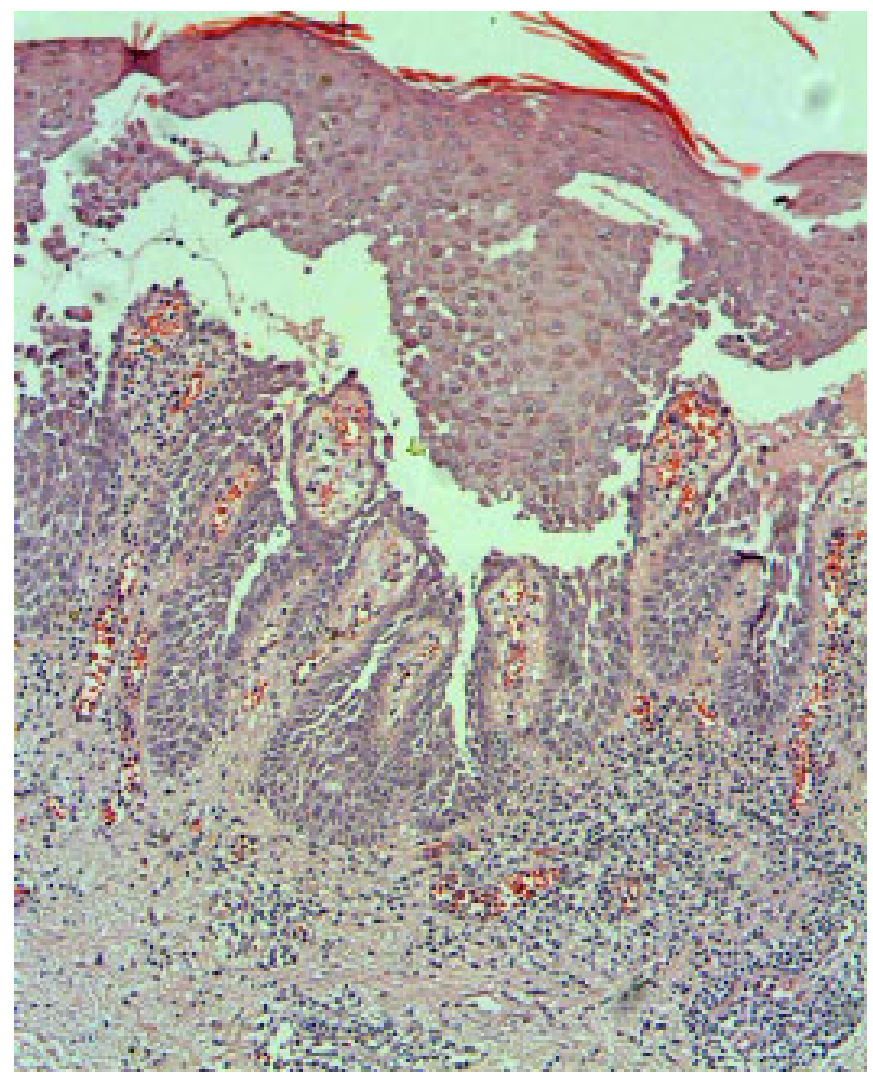

Fig. 2. Histological examination: intraepidermal clefts both suprabasally and higher in the epidermis, characteristic acantholysis with appearance of a "dilapidated brick wall" HEx 10. 

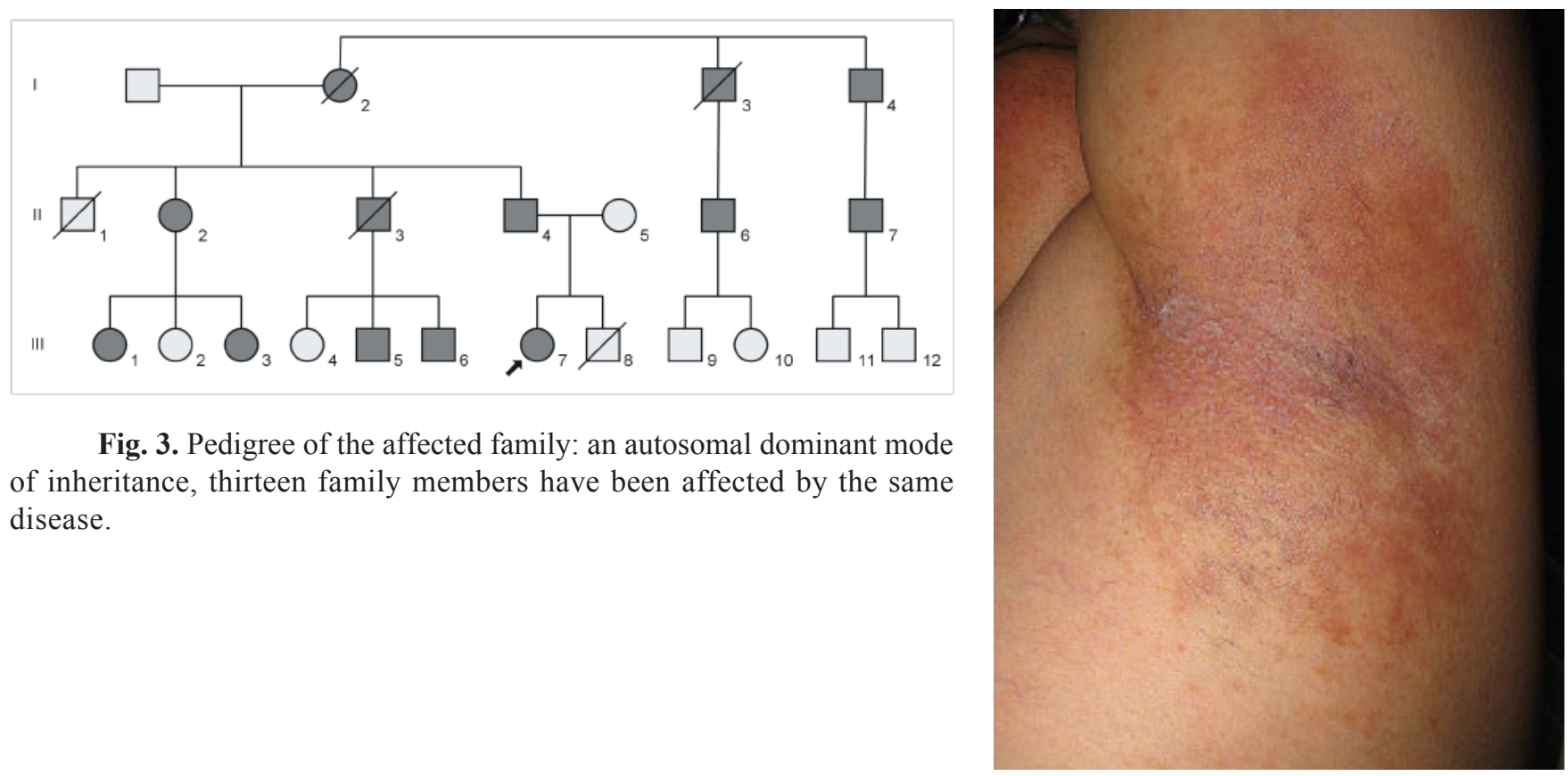

Fig. 3. Pedigree of the affected family: an autosomal dominant mode of inheritance, thirteen family members have been affected by the same disease.

Fig. 4. Postlesional hyperpigmentations in the axillary folds after the treatment with topical pimecrolimus.

\section{REPHERENCES:}

1. Aubert JP, Folchetti G, Berbis P, Magalon G. Surgical treatment of HaileyHailey disease or benign chronic familial pemphigus. Apropos of a case of submammary localization. Ann Chir Plast Esthet. 1993;38(5):568-571.

2. Berth-Jones J, Smith SG, GrahamBrown RA. Benign familial chronic pemphigus (Hailey-Hailey disease) responds to cyclosporin. Clin Exp Dermatol. 1995;20(1):70-72.

3. Burge SM, Millard PR, Wojnarowska F. Hailey-Hailey disease: a widespread abnormality of cell adhesion. Br J Dermatol. 1991;124 (4):329-332.

4. Crotty CP, Scheen SR 3rd, Masson JK, Winkelmann RK. Surgical treatment of familial benign chronic pemphigus. Arch Dermatol. 1981;117(9):540-542.

5. Don PC, Carney PS, Lynch WS, Zaim MT, Hassan MO. Carbon dioxide laserabrasion: a new approach to management of familial benign chronic pemphigus (Hailey-Hailey disease). J Dermatol Surg Oncol. 1987;13(11):11871194.

6. Galimberti RL, Kowalczuk AM, Bianchi O, Bonino MV, Garcia Garcia A. Chronic benign familial pemphigus. Int J Dermatol. 1988;27(7):495-500.

7. Ikeda S, Suga Y, Ogawa H. Successful management of Hailey-Hailey disease with potent topical steroid ointment. J Dermatol Sci. 1993;5(3):205-211.

8. Nicolis G, Tosca A, Marouli O, Stratigos J. Keratosis follicularis and familial benign chronic pemphigus in the same patient. Dermatologica. 1979;159(4):346351.

9. Persic-Vojinovic S, Milavec-Puretic V, Dobric I, Rados J, Spoljar S. Disseminated Hailey-Hailey disease treated with topical tacrolimus and oral erythromycin: Case report and review of the literature. Acta Dermatovenerol Croat. 2006;14(4):253-257.
10. Richter-Hintz D, Megahed M. Disseminated M. Hailey-Hailey. Hautarzt. 2003;54(4):372-374.

11. Schanne R, Burg G, Braun-Falco O. Nosologic relation of dyskeratosis follicularis (Darier) and pemphigus benignus chronicus familiaris (Hailey-Hailey). Position based on a case report Hautarzt. 1985;36(9):504-508.

12. Stallmann D, Schmoeckel C. HaileyHailey disease with dissemination and eczema herpeticatum in therapy with etretinate. Hautarzt. 1988;39(7):454-456.

13. Umar SA, Bhattacharjee P, Brodell RT. Treatment of Hailey-Hailey disease with tacrolimus ointment and clobetasol propionate foam. J Drugs Dermatol. 2004;3(2):200-203.

14. Vaclavinkova V, Neumann E. Vaginal involvement in familial benign chronic pemphigus (Morbus Hailey-Hailey). Acta Derm Venereol. 1982;62(1):80-81.

\section{Address for correspondence:}

Ivelina Yordanova, M.D., Ph.D.

Department of Dermatology and Venereology, Medical University Pleven, 91, General Vladimir Vasov Str. 5800 Pleven, Bulgaria

Tel./fax: +359(64)886 622;

E-mail: ivelina_yordanova@abv.bg; 